\title{
Femtosecond laser near-field ablation from gold nanoparticles
}

\author{
ANTON PLECH ${ }^{1 *}$, VASSILIOS KOTAIDIS ${ }^{1}$, MACIEJ LORENC $^{2}$ AND JOHANNES BONEBERG ${ }^{1}$ \\ ${ }^{1}$ Fachbereich Physik der Universität Konstanz, Universitätsstr. 10, D-78457 Konstanz, Germany \\ ${ }^{2}$ ESRF, BP 220, F-38043, Grenoble Cedex, France \\ *e-mail: anton.plech@uni-konstanz.de
}

$(0$ hort-pulse laser ablation is promising owing to the low threshold for material removal from surfaces. In the laser-ablation process, solid material transforms into a volatile phase initiated by a rapid deposition of energy. Explosive boiling can be one of the mechanisms in which matter is heated close to the critical point. Other pathways of non-thermal excitation will be present for very short laser pulses $^{1}$. Here we observe a different channel of ablation from gold nanoparticles, which takes place below the particle melting point. This process is induced by the optical near-field, a subwavelength field enhancement close to curved surfaces, in particular. Using picosecond $\mathrm{X}$-ray scattering, we can track the temporal and energetic structural dynamics during material ejection from the nanoparticles. This effect will limit any high-power laser manipulation of nanostructured surfaces, such as surface-enhanced Raman measurements ${ }^{2}$ or plasmonics with femtosecond pulses.

Ablation can be characterized as a thermal process, where the material is allowed to thermalize to form a superheated liquid, before ejection sets $\mathrm{in}^{3,4}$. The limit of superheating, which determines the ablation threshold, is related to the spinodal temperature, at which no barrier for vaporization persists. Thermalization can be reached within times exceeding the electron-phonon and phonon-phonon scattering times ${ }^{5}$. For laser pulses shorter than this limit, the explosive boiling will be independent of the pulse length. In addition to the thermal regime, other channels of non-thermal structure modification exist. These will become important for femtosecond excitation. A well-studied example is plasma formation, an important mechanism for transparent media ${ }^{1}$. The so-called dielectric breakdown is caused by multiphoton absorption over the bandgap of dielectrics. Other non-thermal processes are observed in highly excited semiconductors ${ }^{6}$.

Both pathways of material disintegration and ablation will be established at threshold laser fluences considerably above the limits of reversible interaction and material melting $\left(0.4-1.4 \mathrm{~J} \mathrm{~cm}^{-2}\right.$ for 100 fs to $1 \mathrm{~ns}$ pulses ${ }^{1}, 0.25 \mathrm{~J} \mathrm{~cm}^{-2}$ from theoretical calculations ${ }^{7}$, both ablation thresholds on gold films; or $2 \mathrm{~J} \mathrm{~cm}^{-2}$ for dielectric breakdown in fused silica ${ }^{1}$ ). The comparison to values of bulk gold is possible in the present case owing to the relative insensitivity of the interband absorption to geometry. The deposited energy is typically an order of magnitude above the threshold for the melting phase transition in gold. On the other hand, ablation processes have been observed in the vicinity of small metallic structures on a surface, and explained through the optical near-field effect $^{8}$. At edges with high curvature, the evanescent field of the excited structures can reach enhancement factors of several orders of magnitude in comparison with the incident field. Surface-enhanced Raman spectroscopy takes advantage of this effect by amplifying the very weak Raman signal of molecules near corrugated metallic surfaces ${ }^{2}$.

Understanding the fundamental interactions in an atomistic description requires the development of techniques that resolve the dynamics temporally as well as spatially. Ultrafast spectroscopy is a tool to resolve electronic and structural changes accompanied by the laser excitation. However, the spectral fingerprints of electron excitation, altered dielectric environment, particle size change or emerging clusters all tend to overlap in the visible light, making the separation of effects extremely difficult. Work on ultrafast X-ray (and electron) scattering on photo-excited matter has led to the discovery of new modes of phase transitions ${ }^{6,9}$ such as non-thermal melting of densely excited semiconductors. Scattering experiments resolved the molecular rearrangements of photo-induced phase transitions with ångström precision, as in the case of bistable charge-transfer compounds ${ }^{10}$. Picosecond photo-electron spectroscopy has given insight into the structure of ejected material from a silicon surface ${ }^{11}$.

This study presents an observation of ablation from 38-nm gold nanoparticles suspended in water. The energy transduction for gold nanoparticles as model probes for electron dynamics is very well understood (homogeneous electron excitation, followed by electron-phonon coupling, transfer of heat to the environment $\left.{ }^{12,13}\right)$. Sparser data are available on the ionic dynamics; however, it is commonly agreed that most of the structural dynamics observed for particles larger than 5-10 $\mathrm{nm}$ are well within the continuum description ${ }^{14-16}$. A combination of time-resolved $\mathrm{X}$-ray techniques allows us to render a complete picture of the structural rearrangement of the particle crystalline structure and shape, and the interaction with the water phase. The techniques are explained in more detail in the Supplementary Information. 


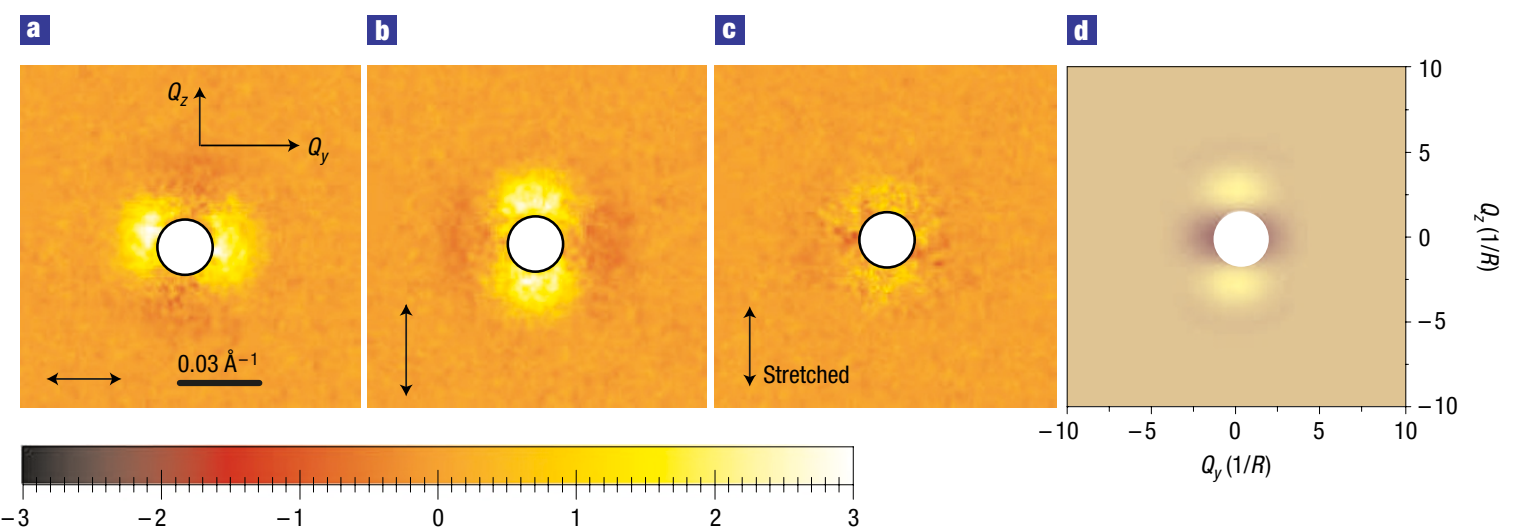

Figure 1 Difference photosignal $\Delta S\left(Q_{y}, Q_{z}\right)$ on the CCD detector in a false colour plot for a $1 \mu$ s delay. The X-ray beam and laser beams are propagating in the $x$ direction. a, Excitation with 100 -fs pulses of $0.0135 \mathrm{~J} \mathrm{~cm}^{-2}$, laser polarization is horizontal; $\mathbf{b}$, the same, but laser polarization is vertical; $\mathbf{c}$, the same, but polarization is vertical and the pulse is stretched to $970 \mathrm{fs}$; $\mathbf{d}$, simulation of the scattering pattern with an oblate particle shape and a size reduction of the $z$-axis (parallel to $Q_{z}$ and parallel to the laser polarization) of $21 \%$. The central circle marks the shadowed region from the beam block.

As length scales are separated in reciprocal space according to the scattering vector $Q=4 \pi / \lambda \cdot \sin (2 \Theta / 2)$, with the scattering angle $2 \Theta$ and X-ray wavelength $\lambda$, the long-range order of the crystalline lattice is recorded at the Bragg peaks. These reflect heating and particle melting ${ }^{16}$. Pressure transients of the water phase are seen in a broad range of scattering angles, $1 \AA^{-1}<Q<$ $5 \AA^{-1}$. They reflect the pair correlation change in water, which stems from pressure variations caused by the expansion of vapour bubbles close to the hot particles ${ }^{17}$. The size and shape of the particles are measured by means of small-angle X-ray scattering (SAXS), which represents a Fourier transform of the shape of the objects. The difference $\Delta S(Q, \tau)$ in the scattering cross-section after laser excitation compared with the native sample has been recorded at the time-resolving beamline ID09B at the European Synchrotron Radiation Facility in Grenoble, France ${ }^{18}$. While laser pulses from a Ti:Sa femtosecond amplifier system excite the gold nanoparticle solution, the synchronized 100-ps X-ray pulses probe the structure as a function of $Q$, laser fluence and laser-to-X-ray delay $\tau$.

Figure 1a-c shows the difference intensity distributions in the SAXS region on the charge-coupled-device (CCD) detector as a function of $Q=\left(Q_{y}, Q_{z}\right)$. The form factor of a sphere is a radial symmetric function of $Q$ with a central maximum, whose width scales with the inverse radius ${ }^{19}$. We observe difference scattering patterns, which lack the isotropy of the distribution within a narrow range of laser fluence for the shown delay of $1 \mu$ s. However, the anisotropy is already found in the earliest measured delay, 150 ps. By $\tau=1 \mu$ s the structural transients of lattice heating and water excitation (including the boiling of the water shell at the particle surface, see Supplementary Information) have decayed out and the final state is observed, whereas the particles are still aligned. The anisotropy (two poles of positive $\Delta S$ signal) is aligned with the laser field vector as seen in Fig. $1 \mathrm{~b}$ after rotating the laser polarization by $90^{\circ}$. The positive intensity is a signature of broadening of the form factor, that is, a reduction of the radius in that direction. In addition, the patterns stem from a direct interaction with the laser field, as the stretching of the laser pulses from 100 to $970 \mathrm{fs}$ leads to a loss of the anisotropy, with hardly any signal observed. At higher fluence, a general reduction in scattering at low $Q$ indicates further photo-modification. It is possible to simulate the measured $\Delta S$ by assuming that the initial spherical gold particles are modified to rotational ellipsoids with the axis parallel to the laser polarization shortened by $21 \%$, that is, a reduction of the particle volume through mass removal at the poles of the sphere (calculation in Fig. 1d).

It is particularly instructive to relate the shape transformation to other observed structural transients on the picosecond timescale (Fig. 2). It is found ${ }^{16}$ (see Supplementary Information) that at low fluence the gold lattice heats up and expands within the temporal resolution of $100 \mathrm{ps}$, followed by a subnanosecond cooling step. The lattice heating is also reflected in the reduction of Bragg intensity due to thermal motion, by the so-called Debye-Waller factor (DWF). In Fig. $2 a$ the measured intensity agrees with the DWF up to a fluence of $0.009 \mathrm{~J} \mathrm{~cm}^{-2}$. At higher fluence, a further reduction is visible, whereas at $0.0155 \mathrm{~J} \mathrm{~cm}^{-2}$ the Bragg scattering vanishes completely, which marks the complete particle melting. Looking at the anisotropy (Fig. 2b) the particle size reduction starts well below the particle melting and reaches a maximum between 0.009 and $0.012 \mathrm{~J} \mathrm{~cm}^{-2}$. The anisotropy vanishes when the melting transition is crossed.

Consequently, the mass removal from the poles of the sphere is already initiated when the particles are still in the solid state. The size reduction is reflected by the Bragg intensity loss before the melting transition. The reduction persists to high fluence, but only the anisotropy disappears. A preservation of the anisotropy is bound to the existence of a solid particle, whereas the liquid particles relax to a sphere, driven by the surface tension.

The shape change is interpreted as surface ablation from the particles through the near-field effect of the laser pulse interacting with a sphere. The calculation of the local electrical field according to the Mie theor $y^{20}$ is shown in Fig. 3. It shows that the input field from the laser pulse is amplified through polarization of the nanoparticle and leads to a strongly enhanced field distribution at the poles of the particle in the shape of a dipolar $\mathrm{coil}^{8}$. The field vector is pointing vertically with respect to the surface. The enhancement factor at the poles amounts to a factor of 11.3, which translates to an electric field of 9.6-12.3 $\mathrm{V} \mathrm{nm}^{-1}$ at the surface ${ }^{21}$ (within the fluence range, where the anisotropy is observed). These values are consistent with measured thresholds for field ion emission from surfaces ${ }^{21,22}$. The ablation mechanism cannot be of thermal origin for the following reasons. First, the temperature is well known and an order of magnitude below the explosive boiling threshold. Second, the anisotropic material removal is extremely fast, compared with isolated emission of single ions. A possible sublimation 


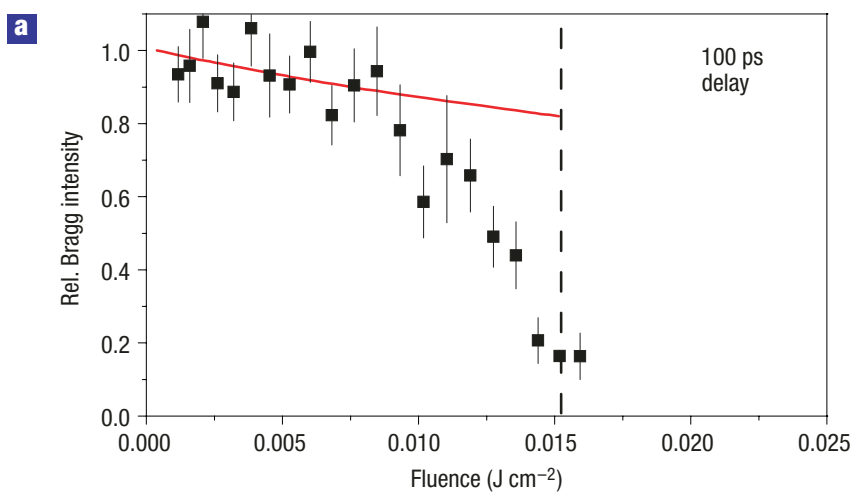

b

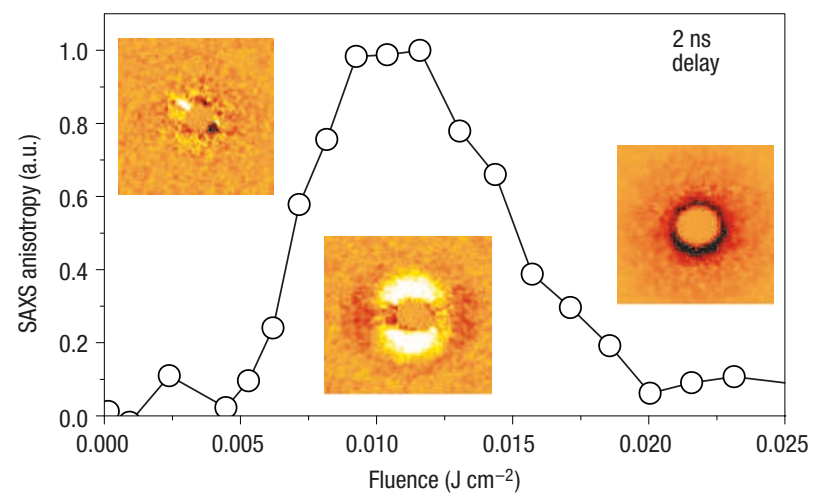

Figure 2 Particle morphology as a function of laser fluence for subnanosecond delays. a, The intensity of the (111) Bragg reflection at 100-ps delay (squares) is plotted together with a calculation of the thermal DWF (full line). The dashed line marks particle melting at $0.0155 \mathrm{~J} \mathrm{~cm}^{-2}$. The error bars are the standard error of the mean value from a set of three repetitions of the experiment. $\mathbf{b}$, The anisotropy of the SAXS patterns as a function of laser fluence as observed for a delay of $2 \mathrm{~ns}$. Insets show false-colour plots of the SAXS patterns in the regions below, within and above the occurrence of anisotropy.

process is rate limited, with emission rates below 1 monolayer per nanosecond ${ }^{23}$. In contrast, we observe a collective process of material ejection with extra cluster emission, which have been observed in ref. 17.

It is furthermore a nonlinear mechanism, as underlined by the pulse-length effect, which reduces the peak field by a factor of six below the emission threshold when the pulses are stretched. Both pulses, compressed and stretched, are still shorter than the electron-phonon coupling time (2-5 ps), which delimits the thermal lattice dynamics. Consequently, a thermal-ablation mechanism should not be sensitive to pulse stretching in the subpicosecond range.

The lower fluence limit may be explained by the onset of bubble nucleation at $0.009 \mathrm{~J} \mathrm{~cm}^{-2}$, which reduces the barrier for ion and cluster separation from the particle, as observed in ablation studies from surfaces ${ }^{24}$. The barrier effect has a stunning analogy to the cage effect in chemical reactions in solution. Above $0.0155 \mathrm{~J} \mathrm{~cm}^{-2}$ the particles melt, which destroys the directional memory of the system as the particles relax to spheres before recrystallization. The presence of ablation is still observed through size reduction of the particles, which is more significant at higher fluence ${ }^{17}$. Interestingly, related effects of laser-induced dichroism have been observed in silver-doped glasses; however, the inversion of the anisotropy points to more complex particle-glass interactions ${ }^{25,26}$.

The experiment shows that a new variant of surface ablation in nanoparticle systems has been observed, which

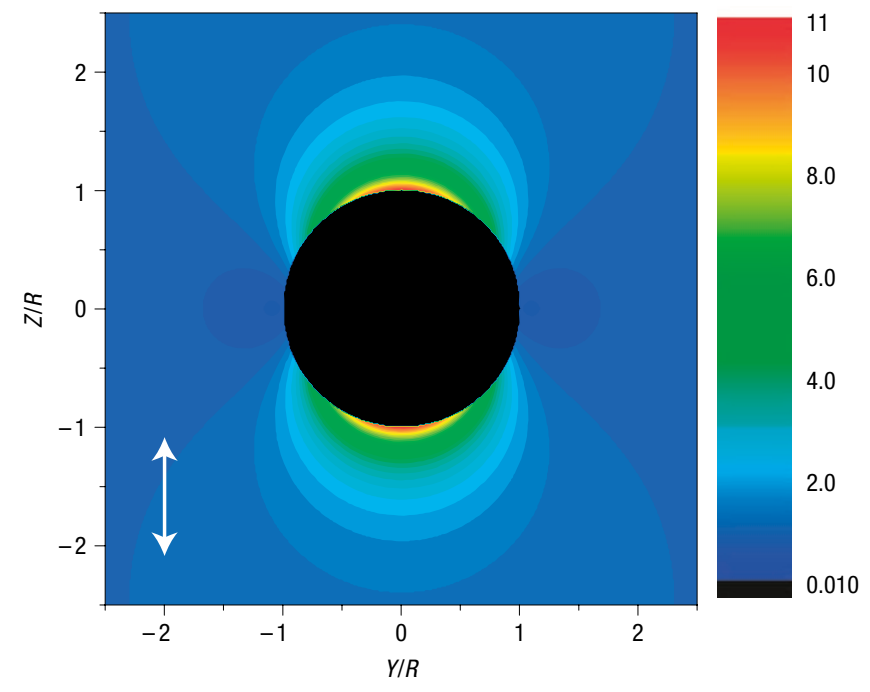

Figure 3 Distribution of the electric field strength of the laser pulse in the vicinity of a spherical particle with a Mie number of 0.397 . The enhancement factor is colour coded and has its peak value of 11.3 close to the particle surface. The arrow marks the electrical polarization vector of the laser field.

is non-thermal in nature, whereas the threshold fluence is considerably lower than would be expected for known mechanisms of ablation with ultrafast lasers. Material ejection is caused by the near-field enhancement on the particles, which is confined to a subwavelength dipolar region. It has been shown that ablation is achievable for laser fluences far below the onset of general catastrophic material deterioration, such as plasma formation or laser-induced explosive boiling ${ }^{1,3,11}$. The limits for the observed anisotropy in the ablation pattern coincide with the reduction of surface barrier due to water vaporization and particle melting. The laser-induced modification of nanoparticle materials is an attractive method for applications in laser-assisted nanomachining, as well as in biomedical applications ${ }^{27,28}$. On the one hand, the effect may be favourable for contactless structuring methods. On the other hand, it may be a drawback when envisioning fully reversible photo-reactions ${ }^{27}$. Surface field enhancements as claimed for surface-enhanced Raman spectroscopy or in plasmonic excitations of nanostructured photonic materials ${ }^{2}$ produce very strong near-fields at the tip of nanostructures. An excitation may lead to ablation, which deteriorates the performance of the materials. Even if the near-field effect for single short laser pulses is weak, material transport would accumulate for repetitive excitation. Consequently, corrugations should also play a role in femtosecond nanomachining. Pulse-length control will be essential to tune reversible versus irreversible interactions of pulsed lasers with nanoscale materials.

\section{METHODS}

Gold nanoparticles of diameter $2 R=38 \mathrm{~nm}$ have been synthesized by the standard Turkevich method ${ }^{29}$. The size distribution is about $15 \%$ for the present batch. The particles are polycrystalline and the shape is spherical.

Laser excitation comes from a $986.2 \mathrm{~Hz}$ Ti:Sa regenerative amplifier (oscillator Mira, Coherent and $1 \mathrm{~mJ}$ amplifier Hurricane, Spectra Physics) after doubling the $800-\mathrm{nm}$ output in a BBO crystal and focusing with a $50 \mathrm{~cm}$ silica lens onto a capillary. The liquid is refreshed within the capillary between the pulses. Pulse stretching can be achieved by the propagation of the 400-nm light through a pair of silica rods. The length of the rods and the group velocity 
dispersion determine the pulse length at the sample position. Here the $100 \mathrm{fs}$ pulse can be stretched to $970 \mathrm{fs}$ after passing $300 \mathrm{~mm}$ of silica.

SAXS has been measured with the pulsed X-ray output of the beamline ID09B at the European Synchrotron Radiation Facility synchronously to the laser excitation. The laser is synchronized to the radiofrequency field of the synchrotron with a precision of $5 \mathrm{ps}$, much better than the X-ray pulse length of $100 \mathrm{ps}$ (ref. 18). The X-ray repetition frequency is reduced to the $986.2 \mathrm{~Hz}$ of the femtosecond amplifier by using a ultrasonic chopper and low filling mode of the synchrotron (16 bunches). For an increased signal-to-noise contrast, the natural bandwidth of the undulator $(2.6 \%)$ was used at a centre wavelength of $0.743 \AA$.

Difference signals $\Delta S(Q, \tau)$ generated after the laser excitation were derived by subtracting two CCD images (the image with the X-ray pulse arrived at the sample at a delay $\tau$ after the laser pulse) from the reference image, in which case the X-ray pulse precedes the laser pulse. The scattering is modelled by the structure function of a spheroid of two equal axes $a=b$ and a variable axis $c$ (ref. 19). The Mie calculation ${ }^{20}$ has been performed with a generalized Mie parameter $\zeta=n \pi \cdot 2 R / \lambda=0.397$, with diameter $2 R=38 \mathrm{~nm}$ of the gold particles, index of refraction $n=1.33$ for water and the laser wavelength $\lambda_{\mathrm{L}}=400 \mathrm{~nm}$ using bulk optical constants for gold. A detailed description of the experiment can be found in the Supplementary Information.

Received 19 August 2005; accepted 21 November 2005; published 18 December 2005.

\section{References}

1. Stuart, B. C. et al. Optical ablation by high-power short-pulse lasers. J. Opt. Soc. Am. B 13, $459-468$ (1996)

2. Haes, A. J. et al. Plasmonic materials for surface-enhanced sensing and spectroscopy. Mater. Res. Soc. Bull. 30, 368-375 (2005).

3. Miotello, A. \& Kelly, R. Critical assessment of thermal models for laser sputtering at high fluences. Appl. Phys. Lett. 67, 3535-3537 (1995).

4. Garrison, B. J., Itina, T. E. \& Zhigilei, L. V. Limit of overheating and the threshold behavior in laser ablation. Phys. Rev. E 68, 041501 (2003).

5. Arbouet, A. et al. Electron-phonon scattering in metal clusters. Phys. Rev. Lett. 90, 177401 (2003).

6. Sokolowski-Tinten, K. et al. Transient states of matter during short pulse laser ablation. Phys. Rev. Lett. 81, 224-227 (1998).

7. Chen, J. K. \& Beraun, J. E. Modelling of ultrashort laser ablation of gold films in vacuum. J. Opt. A 5, $168-173(2003)$

8. Leiderer, P., Bartels, C., König-Birk, J. \& Boneberg, J. Imaging optical near-fields of nanostructures. Appl. Phys. Lett. 85, 5370-5372 (2004).

9. Rousse, A. et al. Non-thermal melting in semiconductors measured at femtosecond resolution. Nature 410, 65-68 (2001).

10. Collet, E. et al. Laser-induced ferroelectric structural order in an organic charge-transfer crystal. Science 300, 612-615 (2003)
11. Glover, T. E. et al. Metal-insulator transitions in an expanding metallic fluid: Particle formation kinetics. Phys. Rev. Lett. 90, 236102 (2003).

12. Link, S. \& El-Sayed, M. A. Shape and size dependence of radiative, non-radiative and photothermal properties of gold nanocrystals. Int. Rev. Phys. Chem. 19, 409-453 (2000).

13. Hu, M. \& Hartland, G. V. Heat dissipation for Au particles in aqueous solution: Relaxation time versus size. J. Phys. Chem. B 106, 7029-7033 (2002).

14. Del Fatti, N., Voisin, C., Chevy, F., Valleé, F. \& Flytzanis, C. Coherent acoustic mode oscillation and damping in silver nanoparticles. J. Chem. Phys. 110, 11484-11487 (1999).

15. Hodak, J. H., Henglein, A. \& Hartland, G. V. Size dependent properties of Au particles: Coherent excitation and dephasing of acoustic vibrational modes. J. Chem. Phys. 111, 8613-8621 (1999).

16. Plech, A., Kotaidis, V., Grésillon, S., Dahmen, C. \& von Plessen, G. Laser-induced heating and melting of gold nanoparticles studied by time-resolved x-ray scattering. Phys. Rev. B 70, 195423 (2004).

17. Plech, A., Kotaidis, V., Lorenc, M. \& Wulff, M. Thermal dynamics in laser excited metal nanoparticles. Chem. Phys. Lett. 401, 565-569 (2005).

18. Schotte, F. et al. in Third-Generation Hard X-ray Synchrotron Radiation Sources (ed. Mills, D.) (Wiley, Berlin, 2002).

19. Guinier, A. \& Fournet, G. Small-angle Scattering of X-rays (Wiley, New York, 1955).

20. Mie, G. Beiträge zur Optik trüber Medien, speziell kolloidaler Metallösungen. Ann. Phys. 4, 377-445 (1908)

21. Gault, B., Vurpillot, F., Bostel, A., Menand, A. \& Deconihout, B. Estimation of the tip field enhancement on a field emitter under laser illumination. Appl. Phys. Lett. 86, 094101 (2005).

22. Chang, C. S., Su, W. B. \& Tsong, T. T. Field evaporation between a gold tip and a gold surface in the scanning tunneling microscope configuration. Phys. Rev. Lett. 72, 574-577 (1994).

23. Miotello, A. \& Kelly, R. Laser-induced phase explosion: New physical problems when a condensed phase approaches the thermodynamic critical temperature. Appl. Phys. A 69, S67-S73 (1999)

24. Tsuji, T., Kakita, T. \& Tsuji, M. Preparation of nano-size particles of silver with femtosecond laser ablation in water. Appl. Surf. Sci. 206, 314-320 (2003).

25. Kaempfe, M., Rainer, T., Berg, K.-J., Seifert, G. \& Graener, H. Ultrashort laser pulse induced deformation of silver nanoparticles in glass. Appl. Phys. Lett. 74, 1200-1202 (1999).

26. Podlipensky, A., Abdolvand, A., Seifert, G. \& Graener, H. Femtosecond laser assisted production of dichroitic 3D structures in composite glass containing Ag nanoparticles. Appl. Phys. A 80, $1647-1652$ (2005).

27. Hirsch, L. R. et al. Nanoshell-mediated near-infrared thermal therapy of tumors under magnetic resonance guidance. Proc. Natl Acad. Sci. USA 100, 13549-13554 (2003).

28. Pitsillides, C. M., Joe, E. K., Wei, X., Anderson, R. R. \& Lin, C. P. Selective cell targeting with light-absorbing microparticles and nanoparticles. Biophys. J. 84, 4023-4032 (2003).

29. Turkevich, J., Stevenson, P. C. \& Hillier, J. A study of the nucleation and growth processes in the synthesis of colloidal gold. Discuss. Faraday Soc. 11, 55-75 (1951)

\section{Acknowledgements}

We wish to thank M. Wulff and Q. Kong for the help with the beamline ID09B and the ESRF for support. Fruitful discussions with P. Leiderer, F. Lang and A. Vogel are acknowledged. This project is funded by the DFG through SFB 513 and the Center for Junior Research Fellows Konstanz.

Correspondence and requests for materials should be addressed to A.P.

Supplementary Information accompanies this paper on www.nature.com/naturephysics.

Competing financial interests

The authors declare that they have no competing financial interests.

Reprints and permission information is available online at http://npg.nature.com/reprintsandpermissions/ 